\title{
Control Method for Sampled-Data Systems with Multiple Channels Based on Deadband-Triggered Scheme
}

\author{
Ying-Ying Liu ${ }^{1}{ }^{1}$ and Yun-kai Chu ${ }^{2}$ \\ ${ }^{1}$ School of Information Engineering, Shenyang University, Shenyang, Liaoning 110044, China \\ ${ }^{2}$ Intelligent Detection and Equipment Department, Shenyang Institute of Automation Chinese Academy of Sciences, \\ Shenyang, Liaoning 110016, China
}

Correspondence should be addressed to Ying-Ying Liu; lyy3636@163.com

Received 2 March 2018; Accepted 15 May 2018; Published 24 June 2018

Academic Editor: Vincenzo Conti

Copyright (c) 2018 Ying-Ying Liu and Yun-kai Chu. This is an open access article distributed under the Creative Commons Attribution License, which permits unrestricted use, distribution, and reproduction in any medium, provided the original work is properly cited.

\begin{abstract}
A new deadband-triggered scheme is proposed to investigate the control problems for sampled-data systems with multiple transmitting channels. Sampled-data systems simultaneously contain continuous-time and discrete-time signals, which make the systems hybrid. In the sampled-data systems with multiple channels, the every state signals are transmitting at different channels. The deadband communication constraint is adopted to reduce the usage of communication resources. When the difference between the previous value and the most present value is lager than a given threshold of deadband, then the node of channels transmits the most present value. Furthermore, by use of Lyapunov functional method and input delay approach, the new stability analysis and stabilization conditions for the sampled-data with multiple channels on the basis of deadband-triggered scheme are proposed. Numerical simulations and experiments show the validity and usefulness of the derived conditions. The proposed deadbandtriggered scheme is beneficial to further reduce the load of the communication data.
\end{abstract}

\section{Introduction}

With the rapid development of digital technology, sensor technology, and the huge performance of these technologies, sampled-data systems has become one of the most widely used systems including industrial processes [1-3]. In this class of system, the object is running in a continuous domain, and the output of the object is a discrete signal measured by a sensor. This makes the controller only rely on discrete sampling signals to monitor objects. So the sampled-data system becomes a hybrid system containing continuous and discrete signals [3-6].

Compared to a generally pure continuous or pure discrete system, the sampled-data system can achieve a more essential description of most of the existing industrial systems or devices. And because of its large use of digital and sensor technologies, this kind of system is more automated and more complex. A typical sampled-data system is networked control system [7-9]. Because of the wide application of the digital hardware technologies, the sampled-data control method has been more important than other control approaches. Recently, some researchers have investigated the sampleddata control [10-12]. However, all the above literature considered that the signals of sampled-data systems are transmitted by one channel. In the actual system, sensor and actuator are sometimes distributed in different areas, the signals require communication through multiple channels, each channel will lead to uncertainties is not the same, and this assumption is not suitable for single channel mode. So it is necessary to study the control problem of sampled-data systems with multiple channels. At present, [13] studied the control problems of the networked control systems based on the channels sharing method. In fact, in many sampled-data systems, the bandwidth of data transmitting channel is limited. Therefore, to avoid the unnecessary waste of communication resources and achieve better performance of sampled-data systems, it is necessary to consider a potential improvement of the traditional sampled-data control methods, namely, the data communication triggered scheme. So far, many results have been obtained about event-triggered control [14-21]. Inspired by 
these results, this paper gives a deadband-triggered scheme, which controls the account of the data transmitting, according to the difference of the present value and the previous value at the deadband-triggered processor. However, the control problems of the sampled-data systems with multiple channel deadband-triggered communication scheme have not been studied in full detail. This motivates the present study.

In response to the above discussion, in this paper, we aim to investigate the stabilization problem for sampled-data systems with multiple communication channels based on deadband-triggered scheme. The organization of this paper is as follows. Section 2 presents the problem of the sampleddata systems under the consideration of deadband-triggered scheme and multiple communication channels. Section 3 presents the stability analysis condition of the sampled-data systems. And, a design method of stabilization for the sampled-data systems is proposed. In Section 4, an example is presented to illustrate the effectiveness of the proposed method. Finally, Section 5 gives some concluding remarks.

Notation. Given a matrix E, $E^{T}$, and $E^{-1}$ denote its transpose and inverse when it exists, respectively. The notation $P>0$ means that $\mathrm{P}$ is real symmetric and positive definite. $I^{n}$ denotes the $n \times n$ identity matrix. In symmetric block matrices, we use an asterisk $*$ to represent a term that is induced by symmetry. $\mathbf{R}^{n}$ and $\mathbf{R}^{m \times n}$ stand for the set of $n$ dimension vectors, $m \times n$ matrices, respectively. The notations throughout this paper are standard. $\operatorname{diag}\{\cdots\}$ denotes a block-diagonal matrix.

\section{Problem Formulation}

2.1. Sampled-Data System with Time-Varying Sampling Intervals. Consider sampled-data system in the form

$$
\dot{x}(t)=A x(t)+B u(t),
$$

where $x(t) \in \mathbf{R}^{n}$ is the system state, $u(t) \in \mathbf{R}^{p}$ is the control input, and $A, B$ are some constant matrices of appropriate dimensions.

The sequence of sampling instants $\left\{t_{k}\right\}_{k=0}^{\infty}$ with $t_{k+1}>t_{k}$ is increasing and divergent with $t_{0}=0$. Without loss generality, it is assumed that $t_{0}=0$ and the first sampling happens at time $t_{0}$. The initial state is given as $x\left(t_{0}\right)=x_{0}$. The sampling interval $T_{k}=t_{k+1}-t_{k}$ is time-varying and its lower bound and upper bound are known:

$$
0<T_{m} \leq T_{k} \leq T_{M}
$$

where $T_{m}$ and $T_{M}$ denote the lower bound of sampling interval and the upper bound of sampling interval, respectively.

We assume that the state of (1) is measurable at $t_{k}$, where the output signal of the controller $u^{\prime}\left(t_{k}\right)$ is determined from the sampled data $x_{i}^{\prime}\left(t_{k}\right)$ and a constant feedback gain $K(K \in$ $\left.\mathbf{R}^{m \times n}\right)$.

2.2. Multiple Communication Channels. In the sampled-data system, it transmits the useful information through multiple channels. As shown in Figure 1, the closed-loop system is composed of a plant, some sensors, some deadband-triggered processors, a controller, and an actuator. The state signals of the plant are sampled at the sensor sides and then released to the communication channels. $x_{i}\left(t_{k}\right)(i=1 \ldots n)$ are the state signals of the plant sampled by the sensors. At the same time, it is assumed that the sensor and sampler are clock-driven, while the controller and actuator are event-driven. As shown in Figure 1, unlike the widely researched state-feedback control system with single channel environment, the signals from sensors are transmitted to the controller via $\mathrm{n}$ different communication channels. The spatially distributed sensors can be synchronized by using some existing approaches such as time synchronization based on the linear consensus algorithm or slow-flooding [19].

In the sampled-data systems, if the sampling interval is too small, the number of data packets will increase quickly, which can lead to an overloaded communication bandwidth. However, fewer sampled signal are must be transmitted for maintaining the running of the controlled systems. As an alternative, this investigation explores a deadband-triggered scheme as a solution to reduce data transmission in the system.

2.3. Deadband-Triggered Communication Scheme. In the sampled-data system (see Figure 1), a deadband-triggered processor (DTP) is set at the every sensor nodes. For the ith channel, the DTP of the sensor to controller is denoted as Deadband $_{i}^{s c}(i=1,2 \ldots n)$. The node does not transfer a new message if the node signal is within the deadband. A number of sampled-data packets will be discarded under the deadband-triggered mechanism, which will make the channel loads reduced greatly. Thus, a good quality of the channel can be possibly ensured.

In Figure 1 , at the sampling instant $t_{k}(k=0,1, \ldots n)$, both the state and the control input signals are processed by deadbands before they are sent to the controller and actuator, respectively. Each state signal $x_{i}\left(t_{k}\right)(i=1,2, \cdots, n)$ is sampled by sensor $i$. Whether or not the sampled signal $x_{i}\left(t_{k}\right)$ should be transmitted is determined by the ith DTP. The mechanism of Deadband ${ }_{i}^{s c}$ is precisely described as follows:

$$
\begin{aligned}
& x_{i}^{\prime}\left(t_{k+1}\right) \\
& \quad= \begin{cases}x_{i}\left(t_{k+1}\right) & \left|x_{i}^{\prime}\left(t_{k}\right)-x_{i}\left(t_{k+1}\right)\right|>\delta_{1 i}\left|x_{i}\left(t_{k+1}\right)\right| \\
x_{i}^{\prime}\left(t_{k}\right) & \left|x_{i}^{\prime}\left(t_{k}\right)-x_{i}\left(t_{k+1}\right)\right| \leq \delta_{1 i}\left|x_{i}\left(t_{k+1}\right)\right|\end{cases}
\end{aligned}
$$

where we denote the input signal of deadband-triggered processor Deadband ${ }_{i}^{s c}$ as $x_{i}\left(t_{k}\right)$ and its output signal as $x_{i}^{\prime}\left(t_{k}\right)$. The deadband node $i$ in Deadband ${ }_{i}^{s c}$ compares the previous value $x_{i}{ }^{\prime}\left(t_{k}\right)$ to the most recent value $x_{i}\left(t_{k+1}\right)$. The data packet is sent over the channels only if the difference value changes more than a given threshold, where $\delta_{1 i}\left|x_{i}\left(t_{k+1}\right)\right|$ is the threshold of DTP Deadband ${ }_{i}^{s c} . \delta_{1 i}$ is the weighting of threshold. 


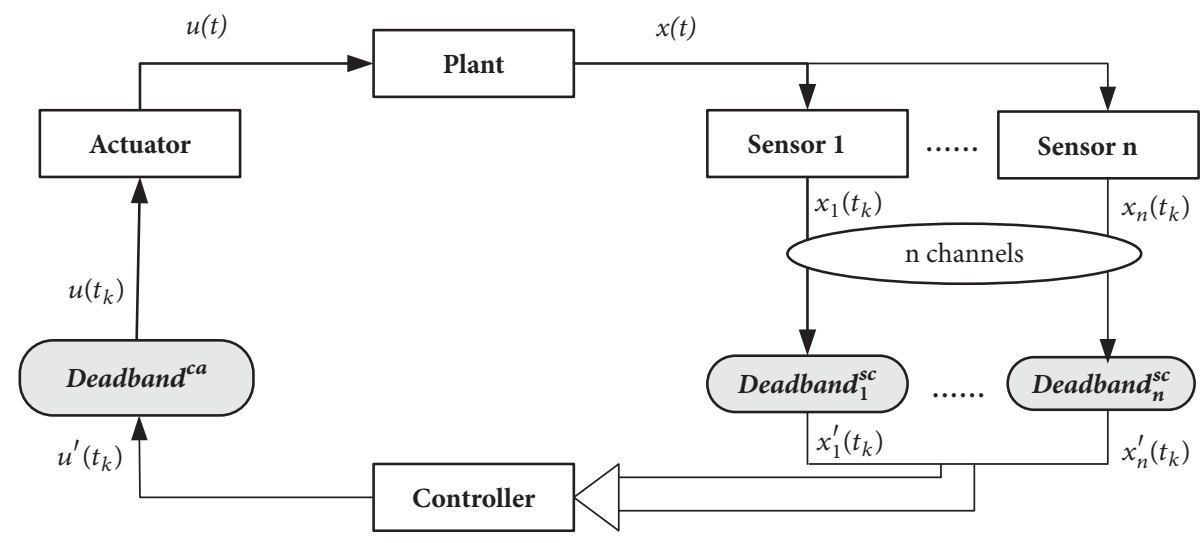

FIGURE 1: Structure of the sampled-data control systems with multiple communication channels.

Remark 1. If the absolute value of the difference between $x_{i}\left(t_{k+1}\right)$ and $x_{i}^{\prime}\left(t_{k}\right)$ is within the threshold of deadband then the signal data is not sent.

Define $\Delta_{1 i}\left(t_{k+1}\right) \in\left[-\delta_{1 i}\left(t_{k+1}\right), \delta_{1 i}\left(t_{k+1}\right)\right]$, then (3) can be included in following equality:

$$
x_{i}^{\prime}\left(t_{k+1}\right)=x_{i}\left(t_{k+1}\right)+\Delta_{1 i}\left(t_{k+1}\right) x_{i}\left(t_{k+1}\right)
$$

Remark 2. Because of the introduction of the deadbandtriggered scheme, some uncertain will be brought to the sampled-data system. We furthermore use (4) to describe deadband mechanism (3) in the model of systems (1). We can clearly see that there exists $x_{i}^{\prime}\left(t_{k+1}\right)=x_{i}\left(t_{k+1}\right)$ when $\Delta_{1 i}\left(t_{k+1}\right)=$ 0 in (4), and there exists $x_{i}^{\prime}\left(t_{k+1}\right)=x_{i}^{\prime}\left(t_{k}\right)$ when $\Delta_{1 i}\left(t_{k+1}\right)=0$ in (4).

For the sake of simplicity, in the following, we use $\delta_{1 i}$ to denote $\delta_{1 i}\left(t_{k+1}\right) . \Delta_{1}\left(t_{k+1}\right)=\operatorname{diag}\left\{\Delta_{11}\left(t_{k+1}\right), \Delta_{12}\left(t_{k+1}\right)\right.$, $\left.\cdots, \Delta_{1 n}\left(t_{k+1}\right)\right\}$. Thus, for the deadband-triggered processor 1 , its input signal and output signal show the following:

$$
x^{\prime}\left(t_{k+1}\right)=\left(I+\Delta_{1}\left(t_{k+1}\right)\right) x\left(t_{k+1}\right)
$$

At the same time, the input signal and the output signal of deadband-triggered processor 2 are $u^{\prime}$ and $u$, respectively. The sampling instants are denoted as $t_{k}, k=0,1, \cdots, \infty$. In the sampled-data system (1), the digital controller is used, and it satisfies the following:

$$
u^{\prime}\left(t_{k}\right)=K x^{\prime}\left(t_{k}\right), \quad k=0,1,2 \ldots
$$

$K$ is the state-feedback gain to be determined later. Following, the relation between the input signal $u^{\prime}$ and the output signal $u$ in deadband-triggered processor 2 can be described as

$$
\begin{aligned}
& u_{j}\left(t_{k+1}\right) \\
& = \begin{cases}u_{j}^{\prime}\left(t_{k+1}\right) & \left|u_{j}\left(t_{k}\right)-u_{j}^{\prime}\left(t_{k+1}\right)\right|>\delta_{2 j}\left|u^{\prime}{ }_{j}\left(t_{k+1}\right)\right| \\
u_{j}\left(t_{k}\right) & \left|u_{j}\left(t_{k}\right)-u_{j}^{\prime}\left(t_{k+1}\right)\right| \leq \delta_{2 j}\left|u^{\prime}{ }_{j}\left(t_{k+1}\right)\right|\end{cases}
\end{aligned}
$$

where $j=1,2, \cdots, m, \delta_{2 j}\left|u_{j}\left(s_{k+1}\right)\right|$ is the threshold of eventtriggered generator $2 . \delta_{2 j}$ is the weighting of threshold.

Similar to (3), (4), then (7) can be included in

$$
u_{j}\left(t_{k+1}\right)=u_{j}^{\prime}\left(t_{k+1}\right)+\Delta_{2 j}\left(t_{k+1}\right) u_{j}^{\prime}\left(t_{k+1}\right)
$$

Define $\Delta_{2}\left(t_{k+1}\right)=\operatorname{diag}\left\{\Delta_{21}\left(t_{k+1}\right), \Delta_{22}\left(t_{k+1}\right), \cdots, \Delta_{2 m}\left(t_{k+1}\right)\right\}$, $\Delta_{2 j}\left(t_{k+1}\right) \in\left[-\delta_{2 j}, \delta_{2 j}\right]$. Combine (4) and (8); the control input signal can be written as

$$
\begin{aligned}
u(t)=\left(I+\Delta_{2}\left(t_{k}\right)\right) K\left(I+\Delta_{1}\left(t_{k}\right)\right) x\left(t_{k}\right) & \\
& t_{k} \leq t<t_{k+1},
\end{aligned}
$$

where $I$ denotes the identity matrix of appropriate dimensions. $\left|\Delta_{1 i}\left(t_{k}\right)\right| \leq \delta_{1 i}$ and $\left|\Delta_{2 j}\left(t_{k}\right)\right| \leq \delta_{2 j}$. For the sake of simplicity, it is assumed that $\delta_{1 i}=\delta_{1}$ and $\delta_{2 j}=\delta_{2}$. Then, it is not difficult to get $\Delta_{1_{i}}\left(t_{k}\right) \in\left[-\delta_{1}, \delta_{1}\right]$ and $\Delta_{2_{j}}\left(t_{k}\right) \in\left[-\delta_{2}, \delta_{2}\right]$. In the following, for the sake of simplicity, we denote $\Delta_{1}\left(t_{k}\right)$, $\Delta_{2}\left(t_{k}\right)$ as $\Delta_{1}, \Delta_{2}$, respectively.

For the sampled-data system, the digital control law may be represented as follows by using input delay approach [12]:

$$
\begin{aligned}
& u(t)=\left(I+\Delta_{2}\right) K\left(I+\Delta_{1}\right)\left(x\left(t-\left(t-t_{k}\right)\right)\right) \\
& t_{k} \leq t<t_{k+1},
\end{aligned}
$$

Denote $d(t)=t-t_{k}$; the piecewise-constant control law (10) can be represented as a continuous-time controller with a time-varying piecewise continuous delay:

$$
\begin{aligned}
& u(t)=\left(I+\Delta_{2}\right) K\left(I+\Delta_{1}\right) x(t-d(t)), \\
& t_{k} \leq t<t_{k+1}
\end{aligned}
$$

where $d(t)=t-t_{k}$ is piecewise linear with the derivative $\dot{d}(t)=1$ for $t \neq t_{k}$. It is assumed that $\mathrm{d}(\mathrm{t})$ is bounded that satisfies $T_{m}<d(t)<T_{M}$.

Substituting the controller (11) into the sampled-data systems (1), we can obtain the closed-loop sampled-data system on the basis of deadband-triggered scheme:

$$
\begin{aligned}
& \dot{x}(t)=A x(t)+B(K+\Delta(K)) x(t-d(t)) \\
& \\
& t_{k} \leq t<t_{k+1}
\end{aligned}
$$

where $\Delta(K)=\Delta_{2} K+K \Delta_{1}+\Delta_{2} K \Delta_{1}$. 
Our objective is to find a state-feedback controller $K$, which stabilizes system (1).

\section{Main Results}

\subsection{Stability Analysis for the Sampled-Data}

Control Systems with Multiple Deadband Channels

Theorem 3. For given scalars $T_{m}, T_{M}, \delta_{1}>0, \delta_{2}>0,0 \leq$ $\alpha<1$, and a matrix $K$, the closed-loop sampled-data system (12) is stable if there exist matrices $P=P^{T}>0, Q_{m}=Q_{m}{ }^{T}>$ $0(m=1,2,3), Z_{j}=Z_{j}{ }^{T}>0(\mathrm{j}=1,2,3)$, and $N_{i}, T_{i}, M_{i}, E_{i}$, $L_{i}(i=1-5)$ such that

$$
\Gamma=\left[\begin{array}{ccccc}
\Gamma_{1} & \Gamma_{2} & \mathscr{B}^{T} Z_{1} & \mathscr{B}^{T} Z_{2} & \mathscr{B}^{T} Z_{3} \\
* & \Gamma_{3} & 0 & 0 & 0 \\
* & * & -\frac{1}{T_{M}-T_{m}} Z_{1} & 0 & 0 \\
* & * & * & -\frac{1}{T_{M}} Z_{2} & 0 \\
* & * & * & * & -\frac{1}{T_{M}} Z_{3}
\end{array}\right]<0,
$$

where

$$
\Gamma_{1}=\left[\begin{array}{ccccc}
\Gamma_{11} & \Gamma_{12} & \Gamma_{13} & \Gamma_{14} & \Gamma_{15} \\
* & \Gamma_{22} & \Gamma_{23} & \Gamma_{24} & \Gamma_{25} \\
* & * & \Gamma_{33} & \Gamma_{34} & \Gamma_{35} \\
* & * & * & \Gamma_{44} & \Gamma_{45} \\
* & * & * & * & \Gamma_{55}
\end{array}\right],
$$

$$
\Gamma_{2}=\left[\begin{array}{ccccc}
N_{1} & T_{1} & M_{1} & E_{1} & L_{1} \\
N_{2} & T_{2} & M_{2} & E_{2} & L_{2} \\
N_{3} & T_{3} & M_{3} & E_{3} & L_{3} \\
N_{4} & T_{4} & M_{4} & E_{4} & L_{4} \\
N_{5} & T_{5} & M_{5} & E_{5} & L_{5}
\end{array}\right],
$$$$
\Gamma_{3}=\operatorname{diag}\left\{-\frac{1}{\alpha T_{M}} Z_{1}-\frac{1}{(1-\alpha) T_{M}} Z_{1}\right.
$$

$$
\begin{gathered}
\left.-\frac{1}{T_{M}-T_{m}}\left(Z_{1}+Z_{2}\right)-\frac{1}{T_{M}} Z_{2}-\frac{1}{T_{M}} Z_{3}\right\}, \\
\Gamma_{11}=P A+A^{T} P+\sum_{i=1}^{3} Q_{i}+N_{1}+N_{1}^{T}+L_{1}+L_{1}^{T},
\end{gathered}
$$

$$
\begin{aligned}
& \Gamma_{12}=P(B K+\Delta(K))+N_{2}^{T}-T_{1}+M_{1}-E_{1}+L_{2}^{T}, \\
& \Gamma_{13}=E_{1}+N_{3}^{T}+L_{3}^{T}, \\
& \Gamma_{14}=-M_{1}+N_{4}^{T}+L_{4}^{T}, \\
& \Gamma_{15}=T_{1}-N_{1}+N_{5}^{T}+L_{5}^{T}, \\
& \Gamma_{22}=M_{2}+M_{2}^{T}-T_{2}-T_{2}^{T}-E_{2}-E_{2}^{T}, \\
& \Gamma_{23}=E_{2}+M_{3}^{T}-T_{3}^{T}-E_{3}^{T}, \\
& \Gamma_{24}=-M_{2}+M_{4}^{T}-T_{4}^{T}-E_{4}^{T}, \\
& \Gamma_{25}=T_{2}+N_{2}, \\
& \Gamma_{33}=-Q_{1}+E_{3}+E_{3}^{T}, \\
& \Gamma_{34}=-M_{3}+E_{4}^{T}-L_{3}, \\
& \Gamma_{35}=T_{3}-N_{3}+E_{5}^{T}, \\
& \Gamma_{44}=-Q_{2}-M_{4}-M_{4}^{T},
\end{aligned}
$$$$
\Gamma_{45}=T_{4}-N_{4}-M_{5}^{T}-L_{5}^{T},
$$$$
\Gamma_{55}=-(1-\alpha) Q_{3}+T_{5}-N_{5}+T_{5}^{T}-N_{5}^{T},
$$$$
\mathscr{B}=\left[\begin{array}{lllll}
A & B(K+\Delta(K)) & 0 & 0 & 0
\end{array}\right],
$$

Proof. Consider the following Lyapunov-Krasovskii functional

$$
\begin{aligned}
V(t)= & V_{1}(t)+V_{2}(t)+V_{3}(t)+V_{4}(t)+V_{5}(t) \\
& +V_{6}(t)+V_{7}(t),
\end{aligned}
$$

where

$$
\begin{aligned}
& V_{1}(t)=x^{T}(t) P x(t), \\
& V_{2}(t)=\int_{t-T_{m}}^{t} x(s)^{T} Q_{1} x(s) d s, \\
& V_{3}(t)=\int_{t-T_{M}}^{t} x(s)^{T} Q_{2} x(s) d s, \\
& V_{4}(t)=\int_{t-\alpha d(t)}^{t} x(s)^{T} Q_{3} x(s) d s, \\
& V_{5}(t)=\int_{-T_{M}}^{0} \int_{t+\beta}^{t} \dot{x}^{T}(s) Z_{1} \dot{x}(s) d s d \beta,
\end{aligned}
$$




$$
\begin{aligned}
& V_{6}(t)=\int_{-T_{M}}^{-T_{m}} \int_{t+\beta}^{t} \dot{x}^{T}(s) Z_{2} \dot{x}(s) d s d \beta, \\
& V_{7}(t)=\int_{-T_{M}}^{0} \int_{t+\beta}^{t} \dot{x}^{T}(s) Z_{3} \dot{x}(s) d s d \beta,
\end{aligned}
$$

$P=P^{T}>0, Q_{m}=Q_{m}{ }^{T}>0(m=1,2,3)$, and $Z_{j}=Z_{j}{ }^{T}>0$ $(\mathrm{j}=1,2,3)$.

(1) For $t_{k}<t<t_{k+1}$, calculating the derivative of $V(t)$ with respect to $t$ along the solutions of the system (12) and using Leibniz-Newton formula, it yields that

$$
\begin{aligned}
& \dot{V}(t)=2 x^{T}(t) P \dot{x}(t)+\sum_{i=1}^{3} x^{T}(t) Q_{i} x(t)-x^{T}\left(t-T_{m}\right) \\
& \cdot Q_{1} x\left(t-T_{m}\right)-x^{T}\left(t-T_{M}\right) Q_{2} x\left(t-T_{M}\right)-(1 \\
& -\alpha \dot{d}(t)) x^{T}(t-\alpha d(t)) Q_{3} x(t-\alpha d(t))+\dot{x}^{T}(t) \\
& \cdot\left(T_{M} Z_{1}+h_{12} Z_{2}+T_{M} Z_{3}\right) \dot{x}(t) \\
& -\int_{t-\alpha d(t)}^{t} \dot{x}^{T}(s) Z_{1} \dot{x}(s) d s \\
& -\int_{t-d(t)}^{t-\alpha d(t)} \dot{x}^{T}(s) Z_{1} \dot{x}(s) d s \\
& \quad-\int_{t-T_{M}}^{t-d(t)} \dot{x}^{T}(s)\left(Z_{1}+Z_{2}\right) \dot{x}(s) d s \\
& \quad-\int_{t-d(t)}^{t-\alpha d(t)} \dot{x}^{T}(s) Z_{1} \dot{x}(s) d s \\
& \quad-\int_{t-d(t)}^{t-T_{m}} \dot{x}^{T}(s) Z_{2} \dot{x}(s) d s \\
& \quad-\int_{M}^{t}(t)\left(T_{M} Z_{1}+\left(T_{M}-T_{m}\right) Z_{2}+T_{M} Z_{3}\right) \dot{x}(t) \\
& \quad \int_{t-T_{M}}^{t} \dot{x}^{T}(s) Z_{3} \dot{x}(s) d s \leq 2 x^{T}(t) P(A x(t) \\
& +x_{1} \dot{x}(s) d s \\
& +B(K+\Delta(K)) x(t-d(t)))+\sum_{i=1}^{3} x^{T}(t) Q_{i} x(t)
\end{aligned}
$$

$$
\begin{aligned}
& -\int_{t-T_{M}}^{t} \dot{x}^{T}(s) Z_{3} \dot{x}(s) d s+2 \zeta^{T}(t) N[x(t) \\
& \left.-x(t-\alpha d(t))-\int_{t-\alpha d(t)}^{t} \dot{x}(s) d s\right]+2 \zeta^{T}(t)
\end{aligned}
$$$$
\cdot T[x(t-\alpha d(t))-x(t-d(t))
$$$$
\left.-\int_{t-d(t)}^{t-\alpha d(t)} \dot{x}(s) d s\right]+2 \zeta^{T}(t) M[x(t-d(t))
$$$$
\left.-x\left(t-T_{M}\right)-\int_{t-T_{M}}^{t-d(t)} \dot{x}(s) d s\right]+2 \zeta^{T}(t)
$$$$
\cdot E\left[x\left(t-T_{m}\right)-x(t-d(t))-\int_{t-d(t)}^{t-T_{m}} \dot{x}(s) d s\right]
$$$$
+2 \zeta^{T}(t) L\left[x(t)-x\left(t-T_{M}\right)-\int_{t-T_{M}}^{t} \dot{x}(s) d s\right]
$$$$
\leq \zeta^{T}(t)\left(\Gamma_{1}+\alpha T_{M} N Z_{1}^{-1} N^{T}+(1-\alpha) T_{M} T Z_{1}^{-1} T^{T}\right.
$$$$
+h_{12} M\left(Z_{1}+Z_{2}\right)^{-1} M^{T}+h_{12} E Z_{2}^{-1} E^{T}
$$$$
+T_{M} L Z_{3}^{-1} L^{T}
$$$$
\left.+\mathscr{B}^{T}\left(T_{M} Z_{1}+\left(T_{M}-T_{m}\right) Z_{2}+T_{M} Z_{3}\right) \mathscr{B}\right) \zeta(t)
$$$$
-\int_{t-\alpha d(t)}^{t} \mathscr{H}_{1} Z_{1}^{-1} \mathscr{H}_{1}^{T} d s-\int_{t-d(t)}^{t-\alpha d(t)} \mathscr{H}_{2} Z_{1}^{-1} \mathscr{H}_{2}^{T} d s
$$$$
-\int_{t-T_{M}}^{t-d(t)} \mathscr{H}_{3}\left(Z_{1}+Z_{2}\right)^{-1} \mathscr{H}_{3}{ }^{T} d s
$$$$
-\int_{t-d(t)}^{t-T_{m}} \mathscr{H}_{4} Z_{2}^{-1} \mathscr{H}_{4}^{T} d s-\int_{t-T_{M}}^{t} \mathscr{H}_{5} Z_{3}^{-1} \mathscr{H}_{5}^{T} d s,
$$

where 


$$
\begin{aligned}
& \mathscr{H}_{1}=\zeta^{T}(t) N+\dot{x}^{T}(s) Z_{1} \\
& \mathscr{H}_{2}=\zeta^{T}(t) T+\dot{x}^{T}(s) Z_{1}, \\
& \mathscr{H}_{3}=\zeta^{T}(t) M+\dot{x}^{T}(s)\left(Z_{1}+Z_{2}\right) \text {, } \\
& \mathscr{H}_{4}=\zeta^{T}(t) E+\dot{x}^{T}(s) Z_{2}, \\
& \mathscr{H}_{5}=\zeta^{T}(t) L+\dot{x}^{T}(s) Z_{3},
\end{aligned}
$$

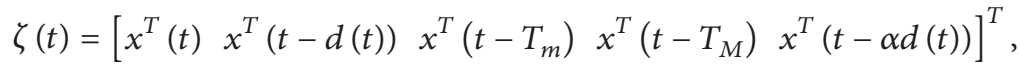

$$
\begin{aligned}
& N=\left[\begin{array}{lllll}
N_{1}^{T} & N_{2}^{T} & N_{3}^{T} & N_{4}^{T} & N_{5}^{T}
\end{array}\right]^{T}, \\
& T=\left[\begin{array}{lllll}
T_{1}^{T} & T_{2}^{T} & T_{3}^{T} & T_{4}^{T} & T_{5}^{T}
\end{array}\right]^{T}, \\
& M=\left[\begin{array}{lllll}
M_{1}^{T} & M_{2}^{T} & M_{3}^{T} & M_{4}^{T} & M_{5}^{T}
\end{array}\right]^{T}, \\
& E=\left[\begin{array}{lllll}
E_{1}^{T} & E_{2}^{T} & E_{3}^{T} & E_{4}^{T} & E_{5}^{T}
\end{array}\right]^{T}, \\
& L=\left[\begin{array}{lllll}
L_{1}^{T} & L_{2}^{T} & L_{3}^{T} & L_{4}^{T} & L_{5}^{T}
\end{array}\right]^{T} .
\end{aligned}
$$

By the Schur complements, combine (13) to obtain $\dot{V}(t)<0$ for all $t_{k}<t<t_{k+1}$.

(2) It is noting that $d\left(t_{k}\right)=t_{k}-s_{k}, \forall k \in N, d\left(t_{k}^{-}\right)=$ $t_{k}-s_{k-1}, \forall k \in N$.

The value of $x$ before and after $t_{k}$ points remains unchanged (since $\mathrm{x}(\mathrm{t})$ is continuous). Then, we have $V_{i}\left(t_{k}^{-}\right)=$ $V_{i}\left(t_{k}\right)(i=1,2,3,5,6,7)$ in Lyapunov-Krasovskii functional (15). Moreover, for $V_{4}(t)$, there exists $V_{4}\left(t_{k}^{-}\right) \geq V_{4}\left(t_{k}\right)$. Thus, $V\left(t_{k}^{-}\right) \geq V\left(t_{k}\right)$ for $k=0,1,2,3, \cdots$.

For $t \in\left[t_{k}, t_{k+1}\right)$, we have

$$
V(t)-V\left(t_{k}\right) \leq 0
$$

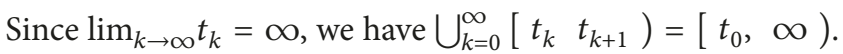
It follows that

$$
V(t)-V\left(t_{0}\right) \leq 0
$$

It shows that $V(t)$ decreases. This completes the proof.

Remark 4. Theorem 3 presents a stability analysis condition of sampled-data systems under deadband-triggered communication scheme, which describes the relations of the deadband parameters and sampling intervals. The given condition is in terms of Linear Matrix Inequality (LMI) when the controller gain $\mathrm{K}$ is given.

3.2. Stabilization for the Sampled-Data Control Systems with Multiple Deadband Channels. This section is devoted to solve the problem of stabilization controller design for sampleddata systems (12). The following theorem presents the conditions for existence of the desired controller.
Theorem 5. Consider the sampled-data system in Figure 1. For given scalars $T_{m}, T_{M}, \delta_{1}>0, \delta_{2}>0,0 \leq \alpha<1$, and $0 \leq \varepsilon_{i}<$ $1(i=1-3)$ the closed-loop system (12) is stable if there exist matrices $X=X^{T}>0, \widetilde{Q}_{i}=\widetilde{Q}_{i}^{T}>0(i=1,2,3), \widetilde{Z}_{j}=$ $\widetilde{Z}_{j}^{T}>0(j=1,2,3), \widetilde{N}_{l}, \widetilde{T}_{l}, \widetilde{M}_{l}, \widetilde{E}_{l}, \widetilde{L}_{l}(l=1-5)$, and $Y$ of appropriate dimensions, such that

$$
\left[\begin{array}{ccc}
\Xi & \widetilde{B} & \widetilde{Y} \\
* & -\frac{1}{\gamma_{1}} I & 0 \\
* & * & -\frac{1}{\gamma_{2}} I
\end{array}\right]<0
$$

where

$$
\begin{aligned}
& \Xi=\left[\begin{array}{ccccc}
\Xi_{1} & \Xi_{2} & A_{L} & A_{L} & A_{L} \\
* & \Xi_{3} & 0 & 0 & 0 \\
* & * & \frac{1}{\bar{\sigma}} \widetilde{Z}_{1}^{*} & 0 & 0 \\
* & * & * & \frac{1}{\sigma} \widetilde{Z}_{2}^{*} & 0 \\
* & * & * & * & \frac{1}{\sigma} \widetilde{Z}_{3}^{*}
\end{array}\right], \\
& \Xi_{1}=\left[\begin{array}{ccccc}
\Xi_{11} & \Xi_{12} & \Xi_{13} & \Xi_{14} & \Xi_{15} \\
* & \Xi_{22} & \Xi_{23} & \Xi_{24} & \Xi_{25} \\
* & * & \Xi_{33} & \Xi_{34} & \Xi_{35} \\
* & * & * & \Xi_{44} & \Xi_{45} \\
* & * & * & * & \Xi_{55}
\end{array}\right],
\end{aligned}
$$




$$
\begin{aligned}
& \Xi_{2}=\left[\begin{array}{cccc}
\widetilde{N}_{1} & \widetilde{T}_{1} & \widetilde{M}_{1} & \widetilde{E}_{1} \\
\widetilde{N}_{2} & \widetilde{T}_{2} & \widetilde{M}_{2} & \widetilde{E}_{2} \\
\widetilde{N}_{3} & \widetilde{T}_{3} & \widetilde{M}_{3} & \widetilde{E}_{3} \\
\widetilde{N}_{4} & \widetilde{T}_{4} & \widetilde{M}_{4} & \widetilde{E}_{4} \\
\widetilde{N}_{5} & \widetilde{T}_{5} & \widetilde{M}_{5} & \widetilde{E}_{5}
\end{array}\right], \\
& \widetilde{Z}_{2}^{*}=\left(\widetilde{Z}_{2}-2 X\right), \\
& \widetilde{Z}_{3}^{*}=\left(\widetilde{Z}_{3}-2 X\right) \text {. } \\
& \Xi_{11}=A X+X A^{T}+\sum_{i=1}^{3} \widetilde{Q}_{i}+\widetilde{N}_{1}+\widetilde{N}_{1}^{T}+\widetilde{L}_{1}+\widetilde{L}_{1}^{T}, \\
& \Xi_{12}=B Y+\widetilde{N}_{2}^{T}-\widetilde{T}_{1}+\widetilde{M}_{1}-\widetilde{E}_{1}+\widetilde{L}_{2}^{T}, \\
& \Xi_{13}=\widetilde{E}_{1}+\widetilde{N}_{3}^{T}+\widetilde{L}_{3}^{T}, \\
& \Xi_{14}=-\widetilde{M}_{1}+\widetilde{N}_{4}^{T}+\widetilde{L}_{4}^{T}, \\
& \Xi_{15}=\widetilde{T}_{1}-\widetilde{N}_{1}+\widetilde{N}_{5}^{T}+\widetilde{N}_{5}^{T}, \\
& \Xi_{22}=\widetilde{M}_{2}+\widetilde{M}_{2}^{T}-\widetilde{T}_{2}-\widetilde{T}_{2}^{T}-\widetilde{E}_{2}-\widetilde{E}_{2}^{T}, \\
& \Xi_{23}=\widetilde{E}_{2}+\widetilde{M}_{3}^{T}-\widetilde{T}_{3}^{T}-\widetilde{E}_{3}^{T}, \\
& \Xi_{24}=-\widetilde{M}_{2}-\widetilde{L}_{2}+\widetilde{M}_{4}^{T}-\widetilde{T}_{4}^{T}-\widetilde{E}_{4}^{T}, \\
& \Xi_{25}=\widetilde{T}_{2}-\widetilde{N}_{2} \\
& \Xi_{33}=-\widetilde{Q}_{1}+\widetilde{E}_{3}+\widetilde{E}_{3}^{T}, \\
& \Xi_{34}=-\widetilde{M}_{3}+\widetilde{E}_{4}^{T}-\widetilde{L}_{3} \text {, } \\
& \Xi_{35}=\widetilde{T}_{3}-\widetilde{N}_{3}+\widetilde{E}_{5}^{T}, \\
& \Xi_{44}=-\widetilde{Q}_{2}-\widetilde{M}_{4}-\widetilde{M}_{4}^{T}, \\
& \Xi_{45}=\widetilde{T}_{4}-\widetilde{N}_{4}-\widetilde{M}_{5}^{T}, \\
& K=Y X^{-1} \text {. } \\
& \widetilde{\Gamma_{1}}=\left[\begin{array}{ccccc}
\Gamma_{11} & \widetilde{\Gamma}_{12} & \Gamma_{13} & \Gamma_{14} & \Gamma_{15} \\
* & \Gamma_{22} & \Gamma_{23} & \Gamma_{24} & \Gamma_{25} \\
* & * & \Gamma_{33} & \Gamma_{34} & \Gamma_{35} \\
* & * & * & \Gamma_{44} & \Gamma_{45} \\
* & * & * & * & \Gamma_{55}
\end{array}\right],
\end{aligned}
$$$$
\Xi_{55}=-(1-\alpha) Q_{3}+\widetilde{T}_{5}-\widetilde{N}_{5}+\widetilde{T}_{5}^{T}-\widetilde{N}_{5}^{T},
$$$$
A_{L}=\left[\begin{array}{lllll}
A X^{T} & B Y & 0 & 0 & 0
\end{array}\right]^{T},
$$$$
\Xi_{3}=\operatorname{diag}\left\{-\frac{1}{\alpha T_{M}} \widetilde{Z}_{1}-\frac{1}{(1-\alpha) T_{M}} \widetilde{Z}_{1}\right.
$$$$
\left.-\frac{1}{T_{M}-T_{m}}\left(\widetilde{Z}_{1}+\widetilde{Z}_{2}\right)-\frac{1}{T_{M}-T_{m}} \widetilde{Z}_{2}-\frac{1}{T_{M}} \widetilde{Z}_{3}\right\},
$$$$
\widetilde{B}=\left[\begin{array}{lllllllllllll}
B^{T} & 0 & 0 & 0 & 0 & 0 & 0 & 0 & 0 & 0 & B^{T} & B^{T} & B^{T}
\end{array}\right]^{T} \text {, }
$$$$
\widetilde{Y}=\left[\begin{array}{lllllllllllll}
0 & Y & 0 & 0 & 0 & 0 & 0 & 0 & 0 & 0 & 0 & 0 & 0
\end{array}\right]^{T} \text {. }
$$$$
\gamma_{2}=\varepsilon_{1}+\varepsilon_{2}+\varepsilon_{3} \delta_{1}^{2} \text {, }
$$$$
\gamma_{1}=\frac{1}{\varepsilon_{1}} \delta_{2}^{2}+\frac{1}{\varepsilon_{2}} \delta_{1}^{2}+\frac{1}{\varepsilon_{3}} \delta_{2}^{2},
$$$$
\widetilde{Z}_{1}^{*}=\left(\widetilde{Z}_{1}-2 X\right) \text {, }
$$

From (13), we have

$$
\begin{aligned}
& \Gamma \\
& =\widetilde{\Gamma}+W_{K}^{T} \Delta_{f} W_{B}+W_{B}^{T} \Delta_{f} W_{K}+\Delta_{g} W_{K}^{T} W_{B} \\
& +W_{B}^{T} W_{K} \Delta_{g}+\Delta_{g} W_{K}^{T} \Delta_{f} W_{B} \\
& +W_{B}^{T} \Delta_{f} W_{K} \Delta_{g} \\
& \leq \widetilde{\Gamma}+\varepsilon_{1} W_{K}{ }^{T} W_{K}+\frac{1}{\varepsilon_{1}} \Delta_{f}{ }^{2} W_{B}{ }^{T} W_{B}+\varepsilon_{2} W_{K}{ }^{T} W_{K} \\
& +\frac{1}{\varepsilon_{2}} \Delta_{g}{ }^{2} W_{B}{ }^{T} W_{B}+\varepsilon_{3} \Delta_{g}{ }^{2} W_{K}{ }^{T} W_{K} \\
& +\frac{1}{\varepsilon_{3}} \Delta_{f}^{2} W_{B}^{T} W_{B} \\
& \leq \widetilde{\Gamma}+\left(\varepsilon_{1}+\varepsilon_{2}+\varepsilon_{3} \delta_{g}^{2}\right) W_{K}^{T} W_{K} \\
& +\left(\frac{1}{\varepsilon_{1}} \delta_{f}^{2}+\frac{1}{\varepsilon_{2}} \delta_{g}^{2}+\frac{1}{\varepsilon_{3}} \delta_{f}^{2}\right) W_{B}^{T} W_{B},
\end{aligned}
$$




$$
\left[\begin{array}{ccc}
\widetilde{\Gamma} & G_{B}^{T} & G_{K}^{T} \\
* & -\frac{1}{\gamma_{1}} I & 0 \\
* & * & -\frac{1}{\varepsilon_{b}} I
\end{array}\right]<0 .
$$

It can be shown that if (13) holds, there exists $\Gamma<0$, that is, $\dot{V}(t)<0$ for all $s_{k}+\tau_{k}<t<s_{k+1}+\tau_{k+1}$.

Define $J=\operatorname{diag}\left\{J_{1}, J_{2}, J_{3}\right\}, J_{1}=\operatorname{diag}\left\{P^{-1}, P^{-1}, P^{-1}, P^{-1}\right.$, $\left.P^{-1}, P^{-1}, P^{-1}, P^{-1}, P^{-1}, P^{-1},\right\}, J_{2}=\operatorname{diag}\left\{Z_{1}^{-1}, Z_{2}^{-1}, Z_{3}^{-1}\right\}$, and $J_{3}=\operatorname{diag}\{I, I\}$.

Pre- and postmultiplying both sides of (26) by $J$ and denoting $X=P^{-1}, \widetilde{N}_{l}=X N_{l} X, \widetilde{T}_{l}=X T_{l} X, \widetilde{M}_{l}=X M_{l} X$, $\widetilde{E}_{l}=X E_{l} X(l=1-5), \widetilde{Q}_{i}=X Q_{i} X(i=1,2,3), \widetilde{Z}_{j}=$ $X Z_{j} X(j=1,2,3)$, and $Y=K X$. Then, we have

$$
\left[\begin{array}{ccc}
\widetilde{\Xi} & \widetilde{B} & \widetilde{Y} \\
* & -\frac{1}{\gamma_{1}} I & 0 \\
* & * & -\frac{1}{\gamma_{2}} I
\end{array}\right]<0,
$$

where

$$
\widetilde{\Xi}=\left[\begin{array}{ccccc}
\Xi_{1} & \Xi_{2} & A_{L} & A_{L} & A_{L} \\
* & \Xi_{3} & 0 & 0 & 0 \\
* & * & -\frac{1}{\bar{\sigma}} Z_{1}^{-1} & 0 & 0 \\
* & * & * & -\frac{1}{\sigma} Z_{2}^{-1} & 0 \\
* & * & * & * & -\frac{1}{\sigma} Z_{3}^{-1}
\end{array}\right],
$$

The conditions in above inequality (27) are not LMI because of the nonlinear terms $Z_{j}^{-1}$. It is noted that $Z_{j}^{-1}=$ $X \widetilde{Z}_{j}^{-1} X(j=1,2,3)$. In order to solve this nonconvex problem, the inequalities in following are needed.

$$
\left(\widetilde{Z}_{m}-X\right) \widetilde{Z}_{m}^{-1}\left(\widetilde{Z}_{m}-X\right) \geq 0 \quad(m=1,2,3),
$$

it is equivalent to

$$
-X \widetilde{Z}_{j}^{-1} X \leq \widetilde{Z}_{j}-2 X \quad(j=1,2,3) .
$$

Then, we can obtain (21). This completes the proof.

Remark 6. By using inequalities (30), the nonlinear terms in (26) are replaced with $\widetilde{Z}_{j}-2 X(j=1,2,3)$, which makes the controller design conditions for the sampled-data systems (12) to be presented in LMI form. From Theorem 5, we can easily obtain the stabilization controller design conditions for the sampled-data systems (12).

\section{Numerical Examples}

Example 1. Consider the following system [22]:

$$
\dot{x}(t)=\left[\begin{array}{cc}
-2 & -0.1 \\
-0.1 & 0.01
\end{array}\right] x(t)+\left[\begin{array}{l}
0.05 \\
0.02
\end{array}\right] u(t)
$$

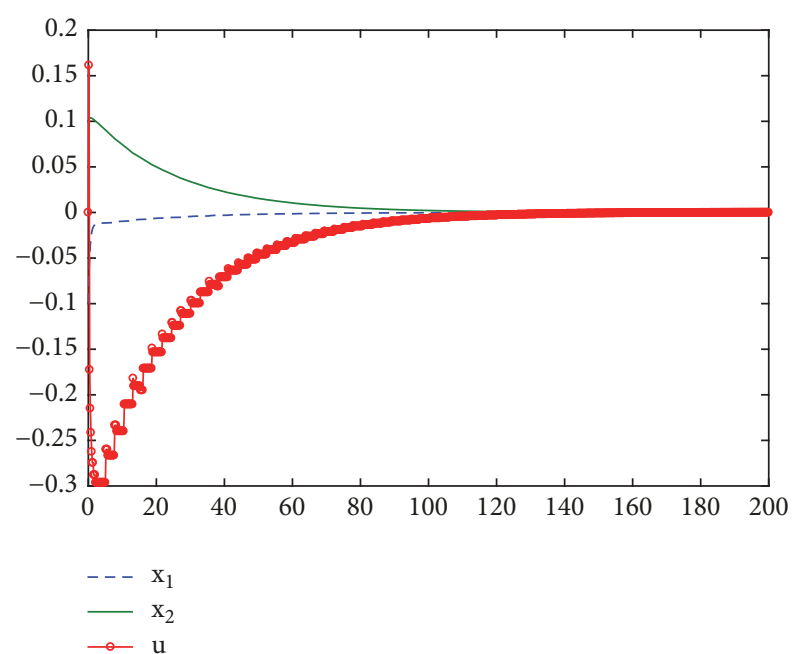

FIGURE 2: State response and control input of the sampled-data system under deadband-triggered scheme.

Choosing $T_{m}=0.10 s, T_{M}=0.20 s, \delta_{1}=0.08$, and $\delta_{2}=0.05$. By using Theorem 5, solving the LMI problem in (21), one can obtain the following solution:

$$
\begin{aligned}
X & =\left[\begin{array}{ll}
10.4216 & -0.0232 \\
-0.0232 & 10.8572
\end{array}\right], \\
Y & =\left[\begin{array}{ll}
11.5574 & -8.9500
\end{array}\right]
\end{aligned}
$$

Hence, the state-feedback controller gain $K=\left[\begin{array}{ll}1.1072 & -0.8220\end{array}\right]$. Figure 2 shows the state response and control input under deadband-triggered scheme, where the initial state of the NCS is $x_{0}=\left[\begin{array}{ll}-0.1 & 0.1\end{array}\right]^{T}$. It can been seen that the closedloop NCS is asymptotically stable with above obtained control gain. It shows the effectiveness of stabilization controller design method proposed in this paper.

Figure 3 shows the transmission of the state signal in two channels $\delta_{1}=0.08$. In channel 1 , for the output signals from deadband processor deadband ${ }_{1}{ }^{s c}$, the previous value is $x_{1}{ }^{\prime}\left(t_{k}\right)$ and the most recent value is $x_{1}{ }^{\prime}\left(t_{k+1}\right)$. In channel 2, for the output signals from deadband processor deadband ${ }_{2}{ }^{s c}$, the previous value is $x_{2}{ }^{\prime}\left(t_{k}\right)$ and the most recent value is $x_{2}{ }^{\prime}\left(t_{k+1}\right)$. In Figure 3, curve output shows the difference between most recent value and the previous value of the output signals from deadband processor deadband ${ }_{1}{ }^{s c}$ and deadband ${ }_{2}{ }^{s c}$. When the curve output is not zero, that is, $x_{i}^{\prime}\left(t_{k+1}\right) \neq x_{i}^{\prime}\left(t_{k}\right)(i=$ $1,2)$, it shows that the state signals are transmitting in the channel 1 and in the channel 2. From Figure 3, one can see that the quantity of data transmission can be reduced and be effectively controlled by adopting the deadband-triggered scheme. Figure 4 shows the transmission of control input at deadband processor deadband ${ }_{1}^{c a}$ for $\delta_{2}=0.05$. It can be seen that the quantity of data transmission can be reduced largely to avoid the heavy of the channels. These have shown that the proposed control approach based on deadband-triggered scheme can guarantee the system stable and effectively reduce the data transmission in the channels. 


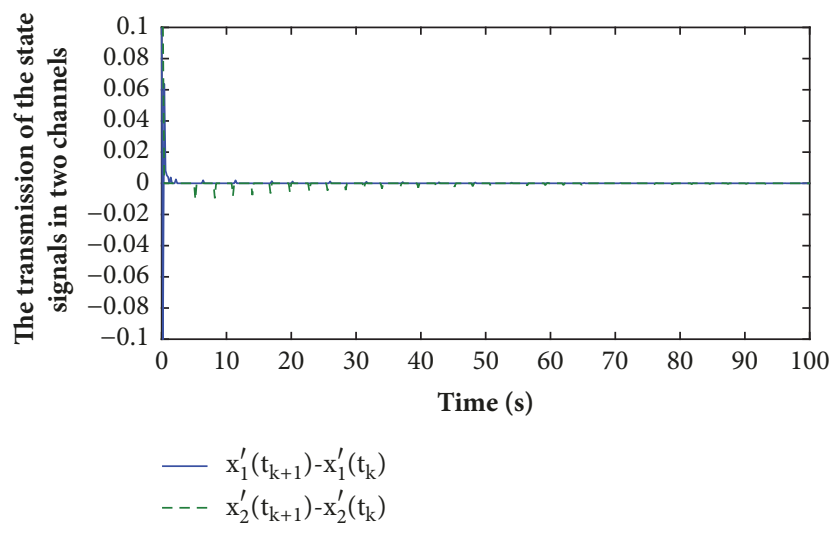

Figure 3: Transmission of state signals under deadband processor 1 for $\delta_{1}=0.08, \delta_{2}=0.05$.

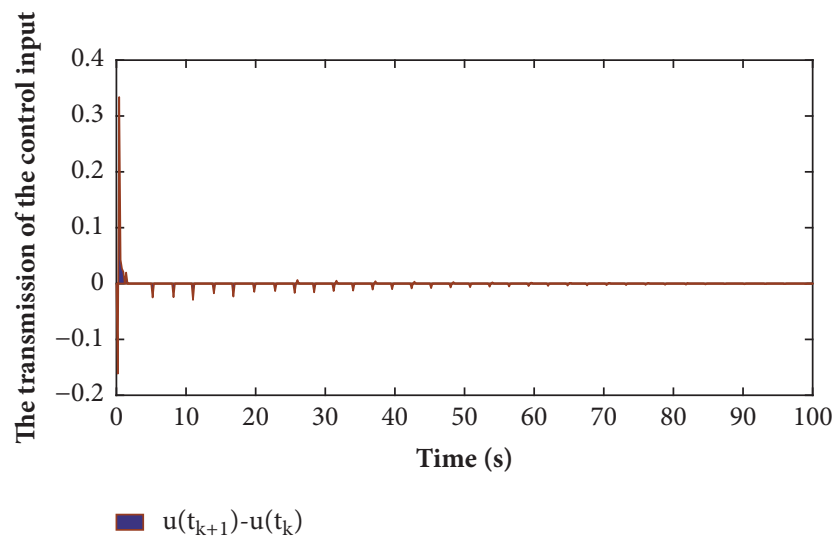

Figure 4: Transmission of control input signals under deadband processor 2 for $\delta_{1}=0.08, \delta_{2}=0.05$.

\section{Conclusions}

This paper investigates the control problem for a sampleddata system with multiple channels, whereby the transmitting of each signal detected by the sensor is depended on the deadband-triggered scheme. By using Lyapunov functional approach and Leibniz-Newton formula, the stability analysis and stabilization methods for the sampled-data system with multiple channels based on deadband-triggered scheme are proposed. Moreover, the proposed stability analysis and stabilization controller design conditions can be presented in terms of linear matrix inequalities (LMIs). A numerical example is used to demonstrate the effectiveness of the proposed methods. From the numerical results, it can be seen that the given method in this paper can guarantee the closed sampled-data system stable, and the proposed deadbandtriggered scheme can significantly reduce the number of data transmitting in the channels.

\section{Data Availability}

The data used to support the findings of this study are available from the corresponding author upon request.

\section{Conflicts of Interest}

The authors declare that they have no conflicts of interest.

\section{Acknowledgments}

This work was supported by the National Nature Science Foundation of China (Grant no. 61603261, Grant no. 61473195, and Grant no. 71601126), the Natural Science Foundation of Liaoning Province (Grant no. 201202156), the Program for Liaoning Excellent Talents in University (LNET) (LJQ2012100), and Program Funded by Liaoning Province Education Administration (Grant no. L2014480).

\section{References}

[1] T. W. Chen and B. A. Francis, Optimal Sampled-Data Control Systems, Springer, 1995.

[2] B. Shen, Z. Wang, and T. Huang, "Stabilization for sampled-data systems under noisy sampling interval," Automatica, vol. 63, pp. 162-166, 2016.

[3] A. Seuret and C. Briat, "Stability analysis of uncertain sampleddata systems with incremental delay using looped-functionals," Automatica, vol. 55, pp. 274-278, 2015.

[4] B. Shen, H. Tan, Z. Wang, and T. Huang, "Quantized/saturated control for sampled-data systems under noisy sampling intervals: a confluent Vandermonde matrix approach," Institute of Electrical and Electronics Engineers Transactions on Automatic Control, vol. 62, no. 9, pp. 4753-4759, 2017.

[5] L. Xie, H. Z. Yang, and F. Ding, "Identification of non-uniformly sampled-data systems with asynchronous input and output data," Journal of The Franklin Institute, vol. 354, no. 4, pp. 19741991, 2017.

[6] L. Hetel, C. Fiter, H. Omran et al., "Recent developments on the stability of systems with aperiodic sampling: an overview," Automatica, vol. 76, pp. 309-335, 2017.

[7] Y.-L. Wang, T.-B. Wang, and Q.-L. Han, "Fault detection filter design for data reconstruction-based continuous-time networked control systems," Information Sciences, vol. 328, pp. 577-594, 2016.

[8] Y.-L. Wang and Q.-L. Han, "Network-based modelling and dynamic output feedback control for unmanned marine vehicles in network environments," Automatica, vol. 91, pp. 43-53, 2018.

[9] L. Su and G. Chesi, "Robust stability analysis and synthesis for uncertain discrete-time networked control systems over fading channels," Institute of Electrical and Electronics Engineers Transactions on Automatic Control, vol. 62, no. 4, pp. 1966-1971, 2017.

[10] Q.-Y. Fan and G.-H. Yang, "Sampled-data output feedback control based on a new event-triggered control scheme," Information Sciences, vol. 414, pp. 306-318, 2017.

[11] V. Suplin, E. Fridman, and U. Shaked, "Sampled-data $H_{\infty}$ control and filtering: nonuniform uncertain sampling," Automatica, vol. 43, no. 6, pp. 1072-1083, 2007.

[12] E. Fridman, "A refined input delay approach to sampled-data control," Automatica, vol. 46, no. 2, pp. 421-427, 2010.

[13] T. B. Wang, C. D. Wu, and Y. L. Wang, "Communication channel sharing-based network-induced delay and packet dropout compensation for networked control systems," IET Control Theory \& Applications, vol. 7, no. 6, pp. 810-821, 2013.

[14] X. M. Zhang and Q. L. Han, "A Decentralized Event-Triggered Dissipative Control Scheme for Systems With Multiple Sensors 
to Sample the System Outputs," IEEE Transactions on Cybernetics, vol. 46, no. 12, pp. 27-45, 2016.

[15] X. F. Wang and M. Lemmon, "On event design in event-triggered feedback systems," Automatica, vol. 47, no. 10, pp. 23192322, 2011.

[16] F.-L. Qu, Z.-H. Guan, D.-X. He, and M. Chi, "Event-triggered control for networked control systems with quantization and packet losses," Journal of The Franklin Institute, vol. 352, no. 3, pp. 974-986, 2015.

[17] J. H. Zhang and G. Feng, "Event-driven observer-based output feedback control for linear systems," Automatica, vol. 50, no. 7, pp. 1852-1859, 2014.

[18] Y.-L. Wang, C.-C. Lim, and P. Shi, "Adaptively adjusted eventtriggering mechanism on fault detection for networked control systems," IEEE Transactions on Cybernetics, vol. 47, no. 8, pp. 2299-2311, 2017.

[19] D. Du, B. Qi, M. Fei, and Z. Wang, "Quantized control of distributed event-triggered networked control systems with hybrid wired-wireless networks communication constraints," Information Sciences, vol. 380, pp. 74-91, 2017.

[20] D. Ye, M. M. Chen, and G. H. Yang, "Distributed adaptive eventtriggered fault-tolerant consensus of multiagent systems with general linear dynamics," IEEE Transactions on Cybernetics, vol. 99, pp. 1-11, 2018.

[21] Y.-L. Wang, P. Shi, C.-C. Lim, and Y. Liu, "Event-Triggered fault detection filter design for a continuous-time networked control system," IEEE Transactions on Cybernetics, vol. 46, no. 12, pp. 3414-3426, 2016.

[22] L. Zha, J.-A. Fang, and J. Liu, "Two channel event-triggering communication schemes for networked control systems," Neurocomputing, vol. 197, pp. 45-52, 2016. 


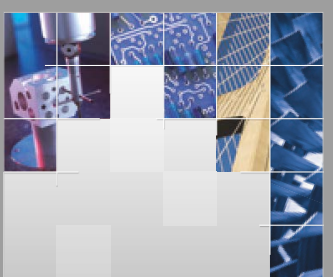

\section{Enfincering}
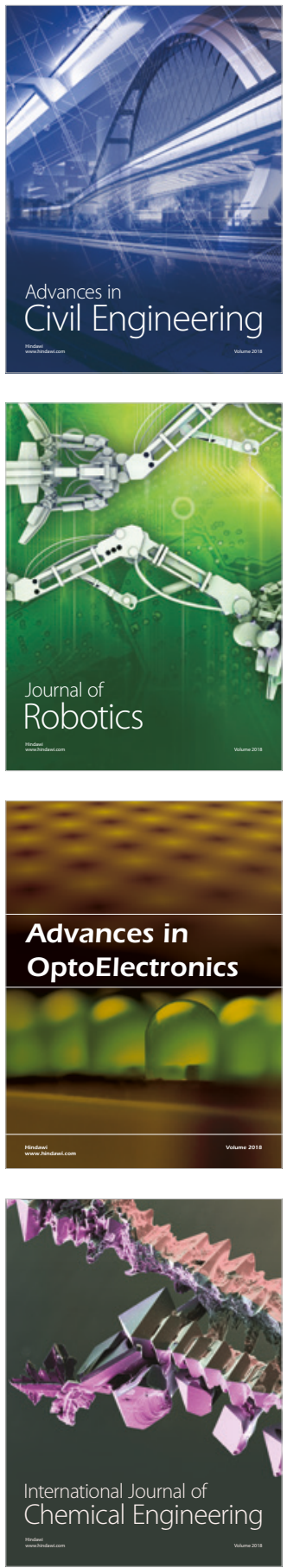

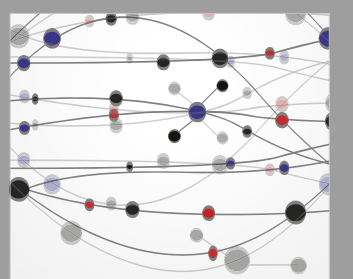

\section{Rotating \\ Machinery}

The Scientific World Journal

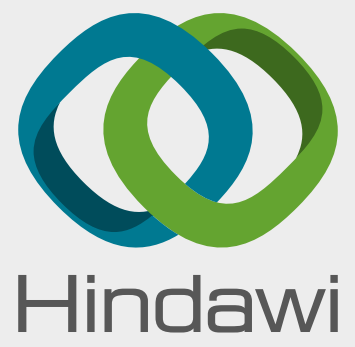

Submit your manuscripts at

www.hindawi.com
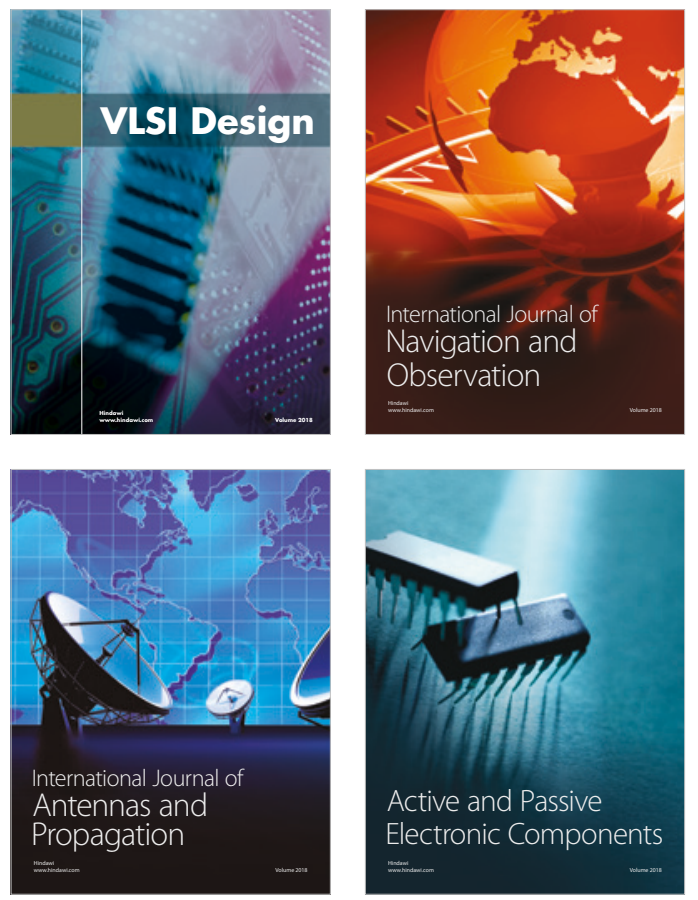
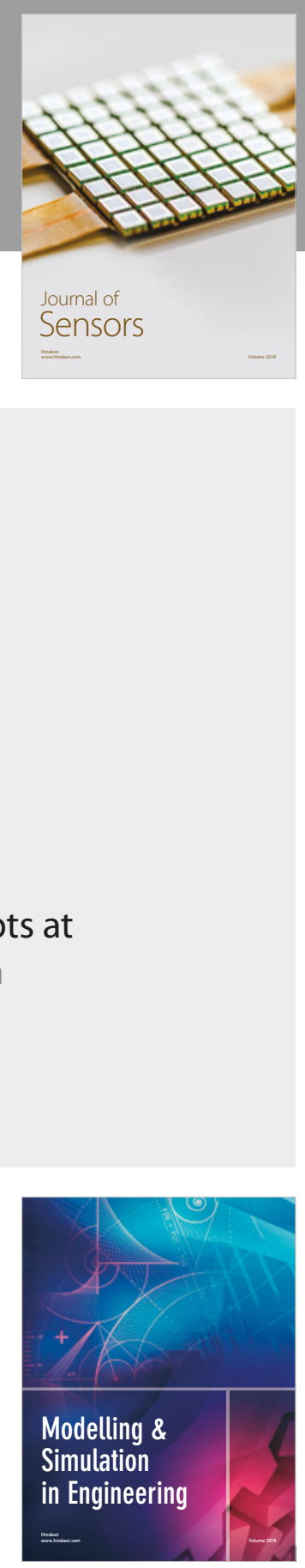

\section{Advances \\ Multimedia}
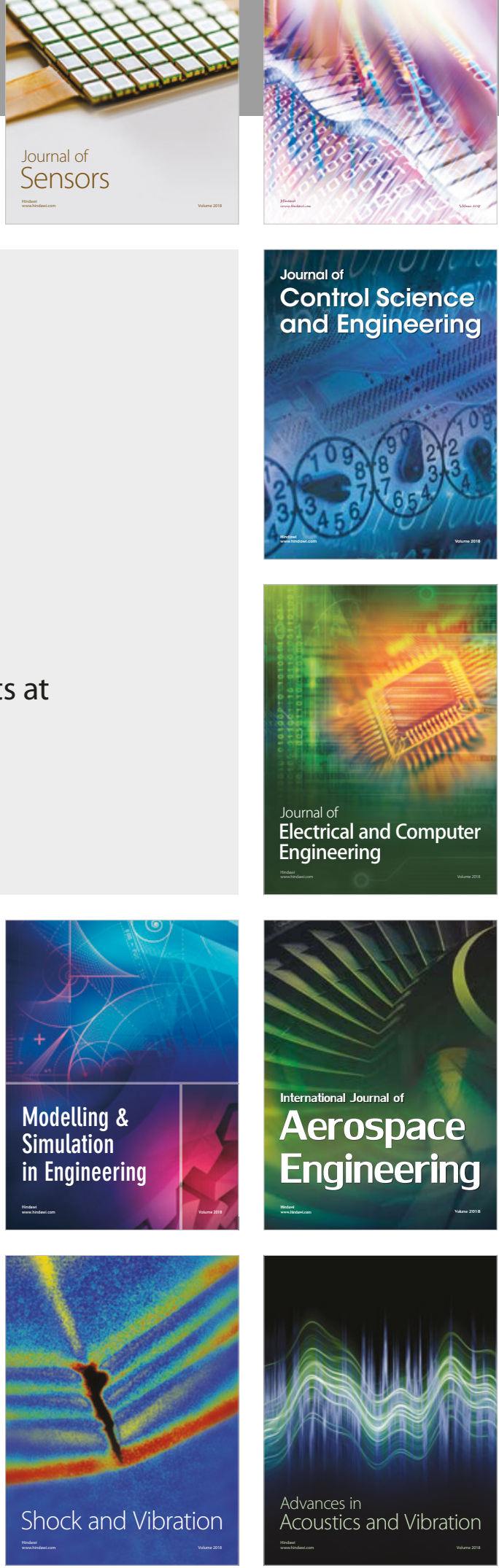